\title{
Partial Discharge Ultraviolet Detection Technology Applied in Enclosed High-voltage Electrical Appliances
}

\author{
Lixin $\mathrm{Ma}^{1, \mathrm{a}}$, Xiaobo Zhou ${ }^{2, \mathrm{~b}}$, Yu Shan ${ }^{3, \mathrm{c}}$ \\ ${ }^{1}$ College of Optics and Electron Information Engineering, University of Shanghai for Science and \\ Technology, Shanghai, 200093, China \\ ${ }^{2}$ College of Optics and Electron Information Engineering, University of Shanghai for Science and \\ Technology, Shanghai ,200093, China \\ ${ }^{3}$ College of Optics and Electron Information Engineering, University of Shanghai for Science and \\ Technology, Shanghai ,200093, China

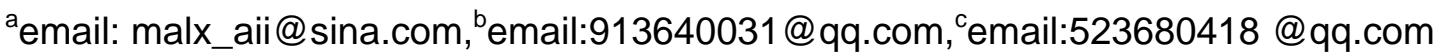

Keywords: UV detection. Wireless communication. Labview.GSM module.

\begin{abstract}
For enclosed high-voltage electrical appliances partial discharge is not easy to be found in inspection and ultraviolet detection equipment cost is high, partial discharge ultraviolet detection system is designed. It is able to monitor the high voltage electrical appliances partial discharge in real time effectively and determine the intensity of the ultraviolet discharge. System communication can satisfy the requirement of real-time application through using the cable transmission to the touch screen, wireless transmission to a nearby server and GSM transmission to the mobile phone. In the actual detection engineering, the preliminary judgment of ultraviolet discharge intensity is based on the analysis of the collected signal. The effectiveness of the proposed system is verified by the experiments.
\end{abstract}

\section{Introduction}

The difficulties to monitor and check and other issues have been existed in enclosed high-voltage electrical appliances. In these devices, due to the surface defects of electrodes and insulation and the damage or aging of wire insulation, the partial discharge of power equipment may happen. If such failure is not handled in time, it is likely to lead to the final breakdown of the insulation failure, and even causes the damage of power equipment. To prevent such accidents, the on-line detection of power equipment partial discharge is very necessary in the condition of not affecting the normal power transmission and transformation.

\section{The principle of UV discharge detection for high voltage electrical}

High pressure equipment for partial discharge occurs, according to different electric field strength, they will produce different forms of discharge. Analysis of the ionization process, electronic constantly get and release energy in the air, Analysis of the spectrum, when the electronic release energy radiates light waves and sound waves, and the spectral emission including ultraviolet, visible and infrared spectrum. While the increase of applied voltage, the radiation spectrum in the UV region of partial discharge will also enhance. UV detection method use photoelectric detector to conversion light signal into electrical signal, and analysis of the electrical signal processing to reflect the situation of partial discharge.

\section{The hardware part of the detection system}

The hardware part of the system includes sensor detection, it includes the data acquisition calculation part and transmission part and display terminal part. Structure is shown in figure 1. 


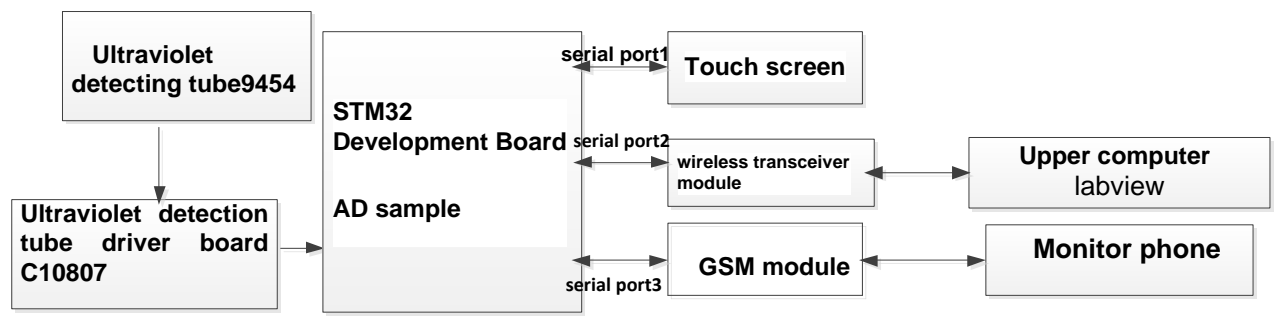

Fig 1 The system structure diagram

\section{The part of Sensor detection}

This article chooses the ultraviolet detection tube is R9454, it is an improved version from R2868, and its wave channel work is UV_C 185-260 nm band of blind area, which effectively avoids the interference of sunlight.

UV detection tube R9454 deals with the driven plate C10807 to get standard square wave signal, however, square wave signal frequency and ultraviolet intensity which discharge produced has a certain relationship.

The part of data acquisition calculation and transmission

The system uses STM32 STM32F103RBT6 chips development board, it collected ultraviolet detection tube to the backwardness of calculating and analyzing the UV signal sent to the display terminal.

AD sampling rules uses rule channel of single sampling mode for the ultraviolet part of the square wave signal, the sampling rate set 239.5 cycle, namely $(239.5+12.5) / 12 \mathrm{~m}=21 \mathrm{mu} \mathrm{s}$ acquisition a time, acquisition of the data input in the memory, through the analysis and calculate of the memory in the acquisition of signal edge cycle to obtain the frequency and the signal.

Transmission parts sent the processed signals via by a serial port 1 to the scene of the touch screen, the signals will also sent to the wireless transceiver module via by a serial port 2 , the upper PC can receive the signals which 1000 m near the machine, the signals sent to the GSM module via by a serial port 3 , and alarm information sent to the phone of monitor with no distance limit.

\section{The part of display terminal}

Display terminal consists of three parts: touch screen, wireless data transceiver module, GSM module.

As shown in figure 2, the touch screen is convenient for the scene security, its image is intuitive, and it is also convenient to set parameter for the touch screen STM32 complete, and so on.

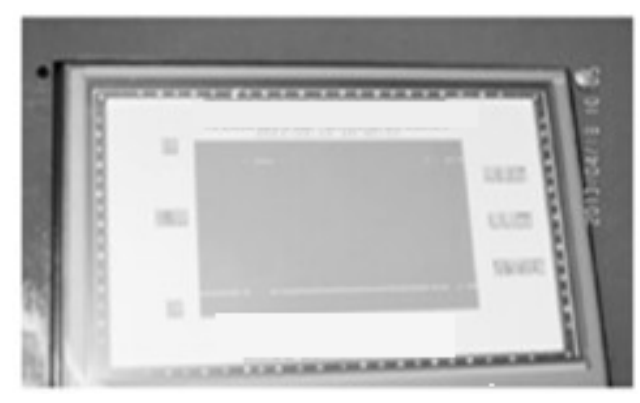

Fig2. Touch screen interface

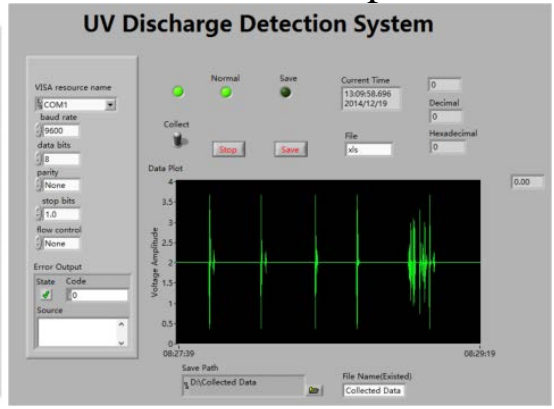

Fig3. The PC interface

As shown in figure 3. The PC can be as a terminal server remote monitoring control room, it can accept data from multiple scenes, the computer is monitoring of the network, the specific number of point to point is determined by computer support serial number, and can keep the collection of UV signal into Excel spreadsheet for convenient query.

The GSM module receives the ultraviolet signal frequency which STM32 development board collected, when the signal frequency is different grades, module send set different short message to the mobile phone, monitor real-time query also can edit text ultraviolet signal frequency, and reaches an alert level, monitoring module can directly call the mobile phone for warning. 


\section{The flow chart of program design}

There are two key problems with program algorithm in the STM32 .one is the signal sampling, another is the frequency calculation. In this paper, the AD collects 500 points in a row, according to each terminal agreement, they were sent to the each serial port to display. Based on the time interval of signal rising along and the next rising along to calculate the frequency, but in order to reduce signal interference of the electromagnetic, all must under the condition of the low level. When continuously appear 10 points high level, it can be rise edge. After processing the standard square wave signal which show in figure 3 . As shown in figure 4 , we can see that the program design flow chart.

\section{The experimental results}

Through the calculation of detection, as shown in figure 5, we find out the relationship between the corresponding discharge strength according to the frequency of UV signal. The waveform is not periodic after the experiment. Therefore, the time high level accounts for in unit time indicates the measured UV intensity. The unit of time is $1 \mathrm{~min}$. Here we use Duty to indicate units. The chart below is the result obtained in the experiment. The abscissa is the current of discharge circuit diagram. The vertical axis is the Duty namely ultraviolet strength. You can see the experimental data graphics and the fitting graphics are not completely coincident, while the error is not large. The current is proportional to the duty cycle. Then we can judge the strength of discharge through the measured data.

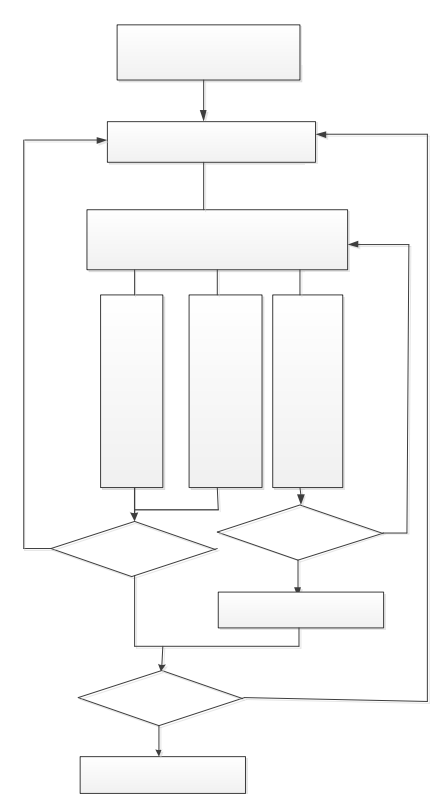

Fig. 4 The flow chart of program

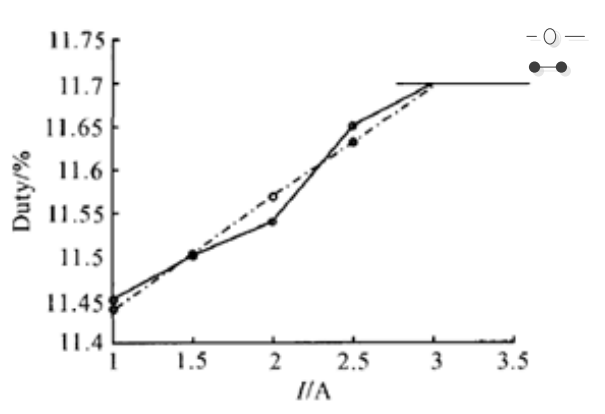

Fig. 5 The experimental results

\section{Conclusion}

In actual situation, the different high voltage equipment have different discharge under different circumstances, therefore, we must make the system in the same condition to detect the different degree of fault discharge intensity. According to the results, the frequency of UV signal that can be artificially divided the grade of the corresponding relations, maintenance and management is convenient. Partial discharge UV detection system is cheap, and it is also convenient for high voltage equipment wireless to monitor. The promotion is significant in the local discharge of the electrical UV detection equipment. 


\section{References}

[1] Chi Dianlin, Ceng Qingli, Sun Lishi. Using UV imaging detection of insulators [J].high voltage engineering, 2006, (2).

[2] Liu Anwen, Tang Wei, Cheng Guangqiang, et al. UV imaging detection technology in the electric power equipment discharge detection on the application of [J].power grid and clean energy, 2013, 29 (3). DOI:10.3969/j.issn.1674-3814.2013.03.010.

[3] Chen Yan, Ye Jianbin, Xie Jianxiang. The UV detection technology used in corona discharge detection [J]. Guangdong electric power, 2008, (9).

[4] Wang Shenghui, Lu Fang Cheng, Li Heming, et al. Development and application of [J]. high voltage electrical equipment of UV imaging detection management system for high voltage apparatus, 2009, (4): 146-149.

[5] Xu Rujun, Lixin Ma, Bo Hu.’'Intelligent High-voltage Discharge Fault Detection and Its Diagnosis Methods Based on ANN,” IPTC, 119-122(2011). 